Communications in Physics, Vol. 25, No. 4 (2015), pp. 309-316

DOI:10.15625/0868-3166/25/4/7306

\title{
ISOTROPIC ROBERTSON-WALKER MODEL UNIVERSE WITH DYNAMICAL COSMOLOGICAL PARAMETER $\Lambda$ IN BRANS-DICKE THEORY OF GRAVITATION
}

\author{
KANGUJAM PRIYOKUMAR SINGH AND MUKUNDA DEWRI \\ Department of Mathematical Sciences, Bodoland University, \\ Kokrajhar, PIN-783370, BTC, Assam, India
}

Received 19 October 2015

Accepted for publication 09 December 2015

E-mail: dewri11@gmail.com

\begin{abstract}
This paper discusses about Robertson-Walker space-time with quadratic equation of state and dynamical cosmological parameter $\Lambda$. Some exact solutions of Einstein's field equations for three cases have been obtained. Physical behaviors of the models are discussed in detail.
\end{abstract}

Keywords: Brans-Dicke theory, dark energy, quadratic equation of state.

\section{INTRODUCTION}

The Brans-Dicke (B-D) theory [1] of gravitation is one of the simplest and best understood scalar-tensor theories. As a result B-D theory has attained significant attention in recent years. Cosmological models in Brans-Dicke theory is discussed by many authors [2-10]. The cosmological and astronomical data obtained from the Supernovae Ia(SNeIa), the cosmic microwave background (CMB) radiation anisotropies, the Large Scale Structure (LSS) and X-ray experiments support the discovery of accelerated expansion of the present day universe [11-19]. The accelerated expansion of universe is due to the presence of dark energy which has positive energy density and adequate negative pressure [20,21]. Chen and Wu [22] considered $\Lambda$ varying as $R^{-2}$, Carvalho and Lima [23] generalized it. Beesham [24], Tiwari [25], Harpreet and Tiwari [26], Kotambkar et al. [27] are some of the authors who studied cosmological model with variable G and $\Lambda$. Nojiri and Odintsov [28], Capozziello [29], Chavanis [30], Sharma and Rantnapal [31], Takisa et al. [32], Feroze and Siddiqui [33] are some of the authors who have discussed about cosmological models with equation of state in quadratic nature. Reddy et al. [34] have obtained Bianchi type-I model with a quadratic equation of state. Ngudelanga [35] has studied about a star with quadratic equation of state. Recently, Adhav et al. [36,37] have obtained some cosmological models with the help of quadratic equation of state.

(C)2015 Vietnam Academy of Science and Technology 
In this paper, we discussed an isotropic cosmological model with perfect fluid in BransDicke theory of gravitation by considering equation of state in quadratic form. In Sec. II we give the field equations and their solution. The discussion is given in Sec. III.

\section{FIELD EQUATIONS AND SOLUTIONS}

Here, we consider the spherically symmetric Robertson Walker metric

$$
d s^{2}=d t^{2}-R^{2}(t)\left[\frac{d r^{2}}{1-k r^{2}}+r^{2}\left(d \theta^{2}+\sin ^{2} \theta d \varphi^{2}\right)\right],
$$

where $k$ is the curvature index which can take values $-1,0,1$.

The Brans-Dicke (B-D) theory of gravity is described by the action

$$
S=\int d^{4} x \sqrt{|g|}\left[\frac{1}{16 \pi}\left(\phi R-\frac{\omega}{\phi} g^{s l} \phi_{, l} \phi_{, s}\right)+L_{m}\right],
$$

where $R$ represents the curvature scalar associated with the 4D metric $g_{i j} ; g$ is the determinant of $g_{i j} ; \phi$ is a scalar field; $\omega$ is a dimensionless coupling constant; $L_{m}$ is the Lagrangian of the ordinary matter component.

The Einstein field equations in the most general form are given by

$$
\begin{aligned}
R_{i j}-\frac{1}{2} R g_{i j}+\Lambda g_{i j} & =\frac{-\kappa}{\phi} T_{i j}-\frac{\omega}{\phi^{2}}\left[\phi_{, i} \phi_{, j}-\frac{1}{2} g_{i j} \phi^{, s} \phi_{, s}\right]-\frac{1}{\phi}\left[\phi_{, i j}-g_{i j} \phi_{; s}^{, s}\right], \\
(3+2 \omega) \phi_{; s}^{, s} & =\kappa T,
\end{aligned}
$$

where $\kappa=8 \pi, \Lambda$ is the cosmological constant, $R_{i j}$ is Ricci-tensor, $g_{i j}$ is metric tensor, $\square \phi=\phi_{;}^{, s}$, and $\phi_{i}$ is the partial differentiation with respect to $x^{i}$ coordinate.

The energy-momentum tensor for the perfect fluid distribution is

$$
T_{i j}=(p+\rho) u_{i} u_{j}-p g_{i j}
$$

with $u_{i}=$ four velocity vector, $p=$ proper density and $\rho=$ proper rest mass density.

Considering a co-moving system, we get

$$
u_{1}=u_{2}=u_{3}=0, u_{4}=1 \text { and } g^{i j} u_{i} u_{j}=1 .
$$

A comma (, ) or semicolon (;) followed by a subscript denotes partial differentiation or a covariant differentiation, respectively. The velocity of light is taken to be unity.

Now for the metric (1) surviving field equations are

$$
\begin{aligned}
\frac{k}{R^{2}}+\frac{\dot{R}^{2}}{R^{2}}+2 \frac{\ddot{R}}{R}-\Lambda & =\frac{-\kappa p}{\phi}-\frac{\omega}{2} \frac{\dot{\phi}^{2}}{\phi^{2}}-2 \frac{\dot{R}}{R} \frac{\dot{\phi}}{\phi}-\frac{\ddot{\phi}}{\phi}, \\
3\left(\frac{k}{R^{2}}+\frac{\dot{R}^{2}}{R^{2}}\right)-\Lambda & =\frac{\kappa \rho}{\phi}+\frac{\omega}{2} \frac{\dot{\phi}^{2}}{\phi^{2}}-3 \frac{\dot{R}}{R} \frac{\dot{\phi}}{\phi} .
\end{aligned}
$$

From Eq. (4), we get

$$
(3+2 \omega)\left[3 \frac{\dot{R} \dot{\phi}}{R}+\ddot{\phi}\right]=\kappa(\rho-3 p)
$$

The energy momentum equation $T_{; j}^{i j}=0$ leads to the form

$$
\dot{\rho}+3 H(\rho+p)=0 \text {. }
$$


We consider [38] ansatz

$$
\Lambda=\beta H^{2}
$$

and equation of state in quadratic form as

$$
p=\alpha \rho^{2}-\rho,
$$

where $\alpha \neq 0$.

From equations (9) and (11), we get

$$
\begin{aligned}
& \rho=R^{-3 \alpha}, \\
& p=\alpha\left[R^{-3 \alpha}\right]^{2}-R^{-3 \alpha} .
\end{aligned}
$$

From equations (6), (7),(8) and (10), we get

$$
3 \frac{k}{R^{2}}+(3-2 \beta) \frac{\dot{R}^{2}}{R^{2}}+3 \frac{\ddot{R}}{R}=\omega\left[\frac{\ddot{\phi}}{\phi}-\frac{1}{2} \frac{\dot{\phi}^{2}}{\phi^{2}}+3 \frac{\dot{R}}{R} \frac{\dot{\phi}}{\phi}\right]
$$

where a dot (.) denotes differentiation with respect to time $t$.

For any cosmological model scale factor $R(t)$ should be known and equation (14) can be integrated by taking the separation constant as zero. So, from (14) we can consider

$$
\begin{aligned}
3 \frac{k}{R^{2}}+(3-2 \beta) \frac{\dot{R}^{2}}{R^{2}}+3 \frac{\ddot{R}}{R} & =0, \\
\frac{\ddot{\phi}}{\phi}-\frac{1}{2} \frac{\dot{\phi}^{2}}{\phi^{2}}+3 \frac{\dot{R}}{R} \frac{\dot{\phi}}{\phi} & =0 .
\end{aligned}
$$

The gravitational variable [39] is given by

$$
G=\left(\frac{4+2 \omega}{3+2 \omega}\right) \frac{1}{\phi}
$$

The anisotropy parameter is given by

$$
\Delta=\frac{1}{3} \sum_{n=1}^{3}\left(\frac{H_{i}-H}{H}\right)^{2} .
$$

Shear scalar is given by

$$
\sigma^{2}=\frac{1}{2}\left[\sum_{i=1}^{3} H_{i}-\frac{1}{3} \Theta^{2}\right] .
$$

II.1. Case I: $k=0$ and $0<\beta<3$

From (15), we get

$$
R=M_{1}\left\{c_{1}(3-\beta)\left(c_{1} t+c_{2}\right)\right\}^{\frac{3}{2(3-\beta)}},
$$

where $M_{1}=2^{\frac{-3}{2(\beta-3)}} 3^{\frac{3}{2(\beta-3)}}$ and $c_{1}, c_{2}$ are constants.

From equation (16), we get

$$
\phi=A^{2} 2^{\frac{9}{(\beta-3)}} 3^{\frac{-3}{(\beta-3)}}\left\{c_{1}(3-\beta)\right\}^{\frac{9}{(\beta-3)}}\left(\frac{\beta-3}{2 \beta+3}\right)^{2}\left(c_{1} t+c_{2}\right)^{\frac{2 \beta+3}{(\beta-3)}},
$$

where $A$ is a constant. 
The gravitational variable is given by

$$
G=\left(\frac{4+2 \omega}{3+2 \omega}\right)\left[A^{2} 2^{\frac{9}{(\beta-3)}} 3^{\frac{-3}{(\beta-3)}}\left\{c_{1}(3-\beta)\right\}^{\frac{9}{(\beta-3)}}\left(\frac{\beta-3}{2 \beta+3}\right)^{2}\left(c_{1} t+c_{2}\right)^{\frac{2 \beta+3}{(\beta-3)}}\right]^{-1} .
$$

From equations (12) and (13), we get

$$
\begin{aligned}
& \rho=\left[M_{1}\left\{c_{1}(3-\beta)\left(c_{1} t+c_{2}\right)\right\}^{\frac{3}{2(3-\beta)}}\right]^{-3 \alpha} \\
& p=\alpha\left[M_{1}\left\{c_{1}(3-\beta)\left(c_{1} t+c_{2}\right)\right\}^{\frac{3}{2(3-\beta)}}\right]^{-6 \alpha}-\left[M_{1}\left\{c_{1}(3-\beta)\left(c_{1} t+c_{2}\right)\right\}^{\frac{3}{2(3-\beta)}}\right]^{-3 \alpha} .
\end{aligned}
$$

Spatial volume is given by

$$
V=\left[M_{1}\left\{c_{1}(3-\beta)\left(c_{1} t+c_{2}\right)\right\}^{\frac{3}{2(3-\beta)}}\right]^{3} .
$$

Hubble's parameter is given by

$$
H=\frac{3}{2 c_{1}(3-\beta)^{2}\left(c_{1} t+c_{2}\right)} .
$$

Scalar expansion is given by

$$
\Theta=\frac{9}{2 c_{1}(3-\beta)^{2}\left(c_{1} t+c_{2}\right)} .
$$

Deceleration parameter is given by

$$
q=-\left(\frac{2 \beta-5}{3}\right)
$$

The anisotropy parameter is given by

$$
\Delta=0 .
$$

Shear scalar is given by

$$
\sigma=0
$$

Cosmological constant is given by

$$
\Lambda=\beta\left[\frac{3}{2 c_{1}(3-\beta)^{2}\left(c_{1} t+c_{2}\right)}\right]^{2} .
$$

II.2. Case II: $k=-1$ and $\beta=3$.

From (15), we

$$
R=M_{2}\left(e^{\frac{c_{4}}{c_{3}}} e^{\frac{t}{c_{3}}}+e^{\frac{-c_{4}}{c_{3}}} e^{\frac{-t}{c_{3}}}\right)
$$

where $M_{2}=\frac{c_{3}}{2}, c_{3}, c_{4}$ are constants.

From equation (16), we get

$$
\phi=c_{5}\left[\left\{\frac{2 e^{2 \frac{c_{4}}{c_{3}}} e^{2 \frac{t}{c_{3}}}+1}{4\left(e^{2 \frac{c_{4}}{c_{3}}} e^{2 \frac{t}{c_{3}}}+1\right)^{2}}\right\}\right]^{2},
$$


where $c_{5}=M_{2}{ }^{-6}$ is a constant.

The gravitational variable is given by

$$
G=c_{5}{ }^{-1}\left(\frac{4+2 \omega}{3+2 \omega}\right)\left[\left\{\frac{2 e^{2 \frac{c_{4}}{c_{3}}} e^{2 \frac{t}{c_{3}}}+1}{4\left(e^{2 \frac{c_{4}}{c_{3}}} e^{2 \frac{t}{c_{3}}}+1\right)^{2}}\right\}\right]^{-2} .
$$

From equations (12) and (13), we get

$$
\begin{aligned}
& \rho=\left[M_{2}\left(e^{\frac{c_{4}}{c_{3}}} e^{\frac{t}{c_{3}}}+e^{\frac{-c_{4}}{c_{3}}} e^{\frac{-t}{c_{3}}}\right)\right]^{-3 \alpha}, \\
& p=\alpha\left[M_{2}\left(e^{\frac{c_{4}}{c_{3}}} e^{\frac{t}{c_{3}}}+e^{\frac{-c_{4}}{c_{3}}} e^{\frac{-t}{c_{3}}}\right)\right]^{-6 \alpha}-\left[M_{2}\left(e^{\frac{c_{4}}{c_{3}}} e^{\frac{t}{c_{3}}}+e^{\frac{-c_{4}}{c_{3}}} e^{\frac{-t}{c_{3}}}\right)\right]^{-3 \alpha} .
\end{aligned}
$$

Spatial volume is given by

$$
V=\left[M_{2}\left(e^{\frac{c_{4}}{c_{3}}} e^{\frac{t}{c_{3}}}+e^{\frac{-c_{4}}{c_{3}}} e^{\frac{-t}{c_{3}}}\right)\right]^{3} .
$$

Hubble's parameter is given by

$$
H=\frac{1}{c_{3}}\left[\frac{1-e^{-2 \frac{c_{4}}{c_{3}}} e^{-2 \frac{t}{c_{3}}}}{1+e^{-2 \frac{c_{4}}{c_{3}}} e^{-2 \frac{t}{c_{3}}}}\right] .
$$

Scalar expansion is given by

$$
\Theta=\frac{3}{c_{3}}\left[\frac{1-e^{-2 \frac{c_{4}}{c_{3}}} e^{-2 \frac{t}{c_{3}}}}{1+e^{-2 \frac{c_{4}}{c_{3}}} e^{-2 \frac{t}{c_{3}}}}\right] .
$$

Deceleration parameter is given by

$$
q=\frac{-1}{c_{3}{ }^{4}}\left[\frac{1+e^{-2 \frac{c_{4}}{c_{3}}} e^{-2 \frac{t}{c_{3}}}}{1-e^{-2 \frac{c_{4}}{c_{3}}} e^{-2 \frac{t}{c_{3}}}}\right] .
$$

The anisotropy parameter is given by

$$
\Delta=0 .
$$

Shear scalar is given by

$$
\sigma=0
$$

Cosmological constant is given by

$$
\Lambda=\frac{3}{c_{3}{ }^{2}}\left[\frac{1-e^{-2 \frac{c_{4}}{c_{3}}} e^{-2 \frac{t}{c_{3}}}}{1+e^{-2 \frac{c_{4}}{c_{3}}} e^{-2 \frac{t}{c_{3}}}}\right]^{2} .
$$


II.3. Case III: $k=1$ and $\beta=3$.

From (15), we get

$$
R=M_{3}\left(e^{\frac{c_{7}}{c_{6}}} e^{\frac{t}{c_{6}}}-e^{\frac{-c_{7}}{c_{6}}} e^{\frac{-t}{c_{6}}}\right)
$$

where $M_{3}=\frac{c_{6}}{2}, c_{6}, c_{7}$ are constants.

From equation (16), we get

$$
\phi=c_{8}\left[\left\{\frac{2 e^{2 \frac{c_{7}}{c_{6}}} e^{2 \frac{t}{c_{6}}}-1}{4\left(e^{2 \frac{c_{7}}{c_{6}}} e^{2 \frac{t}{c_{6}}}-1\right)^{2}}\right\}\right]^{2},
$$

where $c_{8}=M_{3}{ }^{-6}$ is a constant.

The gravitational variable is given by

$$
G=c_{8}^{-1}\left(\frac{4+2 \omega}{3+2 \omega}\right)\left[\left\{\frac{2 e^{2 \frac{c_{7}}{c_{6}}} e^{2 \frac{t}{c_{6}}}-1}{4\left(e^{2 \frac{c_{7}}{c_{6}}} e^{2 \frac{t}{c_{6}}}-1\right)^{2}}\right\}\right]^{-2} .
$$

From equations (12) and (13), we get

$$
\begin{aligned}
& \rho=\left[M_{3}\left(e^{\frac{c_{7}}{c_{6}}} e^{\frac{t}{c_{6}}}-e^{\frac{-c_{7}}{c_{6}}} e^{\frac{-t}{c_{6}}}\right)\right]^{-3 \alpha}, \\
& p=\alpha\left[M_{3}\left(e^{\frac{c_{7}}{c_{6}}} e^{\frac{t}{c_{6}}}-e^{\frac{-c_{7}}{c_{6}}} e^{\frac{-t}{c_{6}}}\right)\right]^{-6 \alpha}-\left[M_{3}\left(e^{\frac{c_{7}}{c_{6}}} e^{\frac{t}{c_{6}}}-e^{\frac{-c_{7}}{c_{6}}} e^{\frac{-t}{c_{6}}}\right)\right]^{-3 \alpha} .
\end{aligned}
$$

Spatial volume is given by

$$
V=\left[M_{3}\left(e^{\frac{c_{7}}{c_{6}}} e^{\frac{t}{c_{6}}}-e^{\frac{-c_{7}}{c_{6}}} e^{\frac{-t}{c_{6}}}\right)\right]^{3} .
$$

Hubble's parameter is given by

$$
H=\frac{1}{c_{6}}\left[\frac{1+e^{-2 \frac{c_{7}}{c_{6}}} e^{-2 \frac{t}{c_{6}}}}{1-e^{-2 \frac{c_{7}}{c_{6}}} e^{-2 \frac{t}{c_{6}}}}\right] .
$$

Scalar expansion is given by

$$
\Theta=\frac{3}{c_{6}}\left[\frac{1+e^{-2 \frac{c_{7}}{c_{6}}} e^{-2 \frac{t}{c_{6}}}}{1-e^{-2 \frac{c_{7}}{c_{6}}} e^{-2 \frac{t}{c_{6}}}}\right]
$$

Deceleration parameter is given by

$$
q=\frac{-1}{c_{6}{ }^{4}}\left[\frac{1-e^{-2 \frac{c_{7}}{c_{6}}} e^{-2 \frac{t}{c_{6}}}}{1+e^{-2 \frac{c_{7}}{c_{6}}} e^{-2 \frac{t}{c_{6}}}}\right] .
$$

The anisotropy parameter is given by

$$
\Delta=0
$$


Shear scalar is given by

$$
\sigma=0
$$

Cosmological constant is given by

$$
\Lambda=\frac{3}{c_{6}{ }^{2}}\left[\frac{1+e^{-2 \frac{c_{7}}{c_{6}}} e^{-2 \frac{t}{c_{6}}}}{1-e^{-2 \frac{c_{7}}{c_{6}}} e^{-2 \frac{t}{c_{6}}}}\right]^{2} .
$$

\section{DISCUSSION}

Here, we have got the following results:

Case I: In this case $R \rightarrow \infty, \phi \rightarrow 0, G \rightarrow \infty, H \rightarrow 0, \Theta \rightarrow 0, V \rightarrow \infty, \Lambda \rightarrow 0$ as $t \rightarrow \infty$. For $t=0$, $R, \phi, G, H, \Theta, V, \Lambda$ become finite. Also, for $\alpha<0, \rho>0$ and $p<0$ which gives positive energy density and negative pressure contributing to the dark energy model with accelerating universe. Here, for $2<\beta<3, q \leq 0$, the deceleration parameter is in the range $-1 \leq q \leq 0$ which is in agreement with the observations made by Riess et al. [12] and Perlmutter et al. [13] i.e. the expansion of the universe is accelerating. Also, $\Delta=0, \sigma=0$ this shows that our model is isotropic and shear free. The value of the cosmological constant for the model is found to be small and positive, which is supported by the observations Garnavich et al. [40, 41] and Schmidt et al. [42]. Case II: In this case $R \rightarrow \infty, \phi \rightarrow 0, G \rightarrow \infty, V \rightarrow \infty$ as $t \rightarrow \infty$ and $H, \Theta, \Lambda$ remains finite for $t \rightarrow \infty$. Again $R, \phi, G, H, \Theta, V, \Lambda$ become finite for $t=0$. Also, for $\alpha<0, \rho>0$ and $p<0$ which gives positive energy density and negative pressure contributing to the dark energy model with accelerating universe. Here, as $t=0$ and $t \rightarrow \infty$, the deceleration parameter is in the range $-1 \leq q \leq 0$ which gives accelerated expansion of the universe. Here, $\Delta=0, \sigma=0$ this shows that our model is isotropic and shear free. The time dependent cosmological constant for the model is small and positive.

Case III: In this case $R \rightarrow \infty, \phi \rightarrow 0, G \rightarrow \infty, V \rightarrow \infty$, as $t \rightarrow \infty$ and $H, \Theta, \Lambda$ become finite for $t \rightarrow \infty$. For $t=0, R, \phi, G, H, \Theta, V, \Lambda$ become finite. Also, for $\alpha<0, \rho>0$ and $p<0$ which gives positive energy density and negative pressure contributing to the dark energy model with accelerating universe. Here, as $t$ varies from 0 to $\infty$, the deceleration parameter is in the range $-1 \leq q \leq 0$ which supports the observations made by Riess et al. [12] and Perlmutter et al. [13] for accelerating universe. Also, $\Delta=0, \sigma=0$ this shows that our model is isotropic and shear free. The time dependent cosmological constant for this model also is small and positive.

\section{REFERENCES}

[1] C. Brans and R. H. Dicke, Phys. Rev. 124 (1961) 925.

[2] N. Banerjee and A. Beesham, Int. J. of Math. Phys. D 6 (1997) 119124.

[3] T. Singh and L. N. Rai, Gen. Rel. Grav. 15 (1983) 815.

[4] L. O. Pimentel, Astrophys. Space Sci. 112 (1985) 175183.

[5] E. A. Azar and N. Riazi, Astrophys. Space Sci. 226 (1995) 15.

[6] T. Etoh et al., Astron. and Astrophys. 325 (1997) 893.

[7] G. P. Singh and A. Beesham, Austral. J. Phys. 52 (1999) 103949.

[8] A. A. Sen, S. Sen, and S. Sethi, Phys. Rev. D 63 (2001) 107501.

[9] D. R. K. Reddy et al., Int. J. Theor. Phys. 46 (2007) 1443.

[10] K. S. Adhav et al., Int. J. Theor. Phys. 48 (2009) 178.

[11] P. Bernardis et al., Nature 404 (2000) 955.

[12] A. G. Riess et al. Astron. J. 116 (1998) 1009. 
[13] S. Perlmutter et al., Astrophys. J. 517 (1999) 565.

[14] U. Seljak et al. Phys. Rev. D 71 (2005) 103515.

[15] P. Astier et al., Astron. Astrophys. 447 (2006) 31.

[16] C. L. Bennett et al. Astrophys. J. Suppl. Ser. 148 (2003) 1.

[17] D. N. Spergel et al., Astrophys. J. Suppl. Ser. 148 (2003) 175.

[18] E. Komatsu et al., Astrophys. J. Suppl. 180 (2009) 330.

[19] J. K. Adelman-McCarthy et al., Astrophys. J. Suppl. Ser. 175 (2008) 297.

[20] T. Padmanabhan, Phys. Rept. 380 (2003) 235.

[21] V. Sahni and A. A. Starobinsky, Int. J. Mod. Phys. D 9 (2000) 373.

[22] W. Chen and Y. S. Wu, Phys. Rev. D 41 (1990) 695

[23] J. C. Carvalho, J. A. Lima, and I. Waga, Phys. Rev. D 46(6) (1992) 2404.

[24] A. Beesham, Gen. Relativ. Grav. 26 (1994) 2.

[25] R. K. Tiwari, The African Review of Physics 9 (2014) 0053.

[26] Harpreet and R. K. Tiwari, IJPMS 5 (2015) $42-46$

[27] S. Kotambkar, G. P. Singh and R. Kelkar, Natural Science 7 (2015) 179-189.

[28] S. Nojiri and S. D. Odintsov, Phys. Rev. D 72 (2005) 023003.

[29] S. Capozziello et al., Phys. Rev. D 73 (2006) 043512.

[30] P. H. Chavanis, Journal of Gravity (2013) 82451; dx.doi.org/10.1155/2013/682451.

[31] R. Sharma and B. S. Ratanpal, Int. J. Mod. Phys. D 22 (2013) 13.

[32] P. M. Takisa, S. D. Maharaj, and S. Ray, Astrophys. Space Sci. 354 (2014) 463.

[33] T. Feroze and A. A. Siddiqui, JKPS 65(6) (2014) 944-947.

[34] D. R. K. Reddy, Astrophys. Space Sci. 357 (2015) 20.

[35] S. A. Ngubelanga et al., Astrophys. Space Sci. 357 (2015) 74.

[36] K. S. Adhav et al., EIJST 4 (2015) 5.

[37] K. S. Adhav et al., Bulg. J. Phys. 42 (2015) 20-28.

[38] I. A. Arbab, Gen. Relativ. Grav. 29 (1997) 61-74.

[39] S. Weinberg, Gravitation and Cosmology, 2nd edn: Wiley, New York, 1972.

[40] P. M. Garnavich et al., Astrophys. J. 493 (1998) L53

[41] P. M. Garnavich et al., Astrophys. J. 509 (1998) 74

[42] B. P. Schmidt et al., Astrophys. J. 507 (1998) 46 